[0212-7199 (2006) 23: 6; pp 288-290] ANALES DE MEDICINA INTERNA Copyright () 2006 ARAN EDICIONES, S.L. AN. MED. INTERNA (Madrid)
Vol. 23, N. ${ }^{\circ}$ 6, pp. 288-290, 2006

\title{
Síndrome de Ballooning "abortado"
}

\author{
B. OBÓN AZUARA, M. R. ORTAS NADAL'1, I. GUTIÉRREZ CÍA, B. VILLANUEVA \\ ANADÓN, A. CÁRCAMO MERINO
}

Servicios de Medicina Intensiva y ${ }^{\prime}$ Cardiología. Hospital Clínico Universitario. Zaragoza

“ABORTED” BALLOONING SYNDROME

\begin{abstract}
RESUMEN
Paciente con antecedente de síndrome de discinesia apical transitoria los tres meses previos, quien tras un cuadro de estrés emocional presenta un episodio de dolor torácico típico, acompañado de aparición de un gradiente dinámico severo en el tracto de salida de ventrículo izquierdo (TSVI), que se normaliza tras la instauración precoz de betabloqueante.
\end{abstract}

PALABRAS CLAVE: Discinesia apical transitoria "abortada". Obstrucción dinámica en tracto de salida de ventrículo izquierdo

\begin{abstract}
We describe a patient with previous transient left ventricular apical balloonig three months ago, who had got a new chest ischemic-like pain associated to an important dynamic left ventricular intracavitary obstruction which disappeared after betablockers treatment.
\end{abstract}

KEY WORDS: "Aborted" transient left ventricular apical ballooning. Dynamic left ventricular intracavitary obstruction.

Obón Azuara B, Ortas Nadal MR, Gutiérrez Cía I, Villanueva Anadón B, Cárcamo Merino A. Síndrome de Ballooning “abortado”. An Med Interna (Madrid) 2006; 23: 288-290.

\section{INTRODUCCIÓN}

El síndrome de discinesia apical transitoria (SDAT) o cardiomiopatía de Takotsubo, es un síndrome de reciente aparición (1) que simula un infarto agudo de miocardio (IAM) con elevación del segmento ST, mínima elevación enzimática y disfunción sistólica transitoria del VI que afecta al ápex cardiaco, en ausencia de enfermedad aterotrombótica en arterias coronarias (2).

Los pacientes son mujeres con mayor frecuencia, postmenopáusicas y los síntomas aparecen típicamente tras un cuadro de estrés emocional; posee un pronóstico generalmente bueno, si bien existen algunos casos de muerte súbita por fibrilación ventricular (3) u otras complicaciones $(1,4)$. Su fisiopatología resulta desconocida: se ha sugerido la posibilidad de un mecanismo patogénico mediado por catecolaminas 1 dado que los síntomas se encuentran precedidos de un estrés físico o emocional severo, si bien parece que la generación de un gradiente dinámico intraventricular transitorio desempeña un papel fundamental en su etiología $(5,6)$. Una disposición geométrica a nivel del o septo interventricular, conllevaría mayor riesgo de desarrollar la obstrucción suabórtica dinámica, que se manifestaría únicamente bajo situaciones de hiperestimulación adrenérgica $(5,6)$.

\section{CASO APORTADO}

Paciente de sexo femenino de 57 años de edad con antecedentes de hipercolesterolemia y ablación de vía accesoria oculta los 5 años previos. Así mismo, hace 3 meses, tras una discusión, ingresó en una UCI de otro centro hospitalario por presentar un episodio de dolor torácico con elevación del segmento ST en derivaciones precordiales e inferiores del electrocardiograma (ECG) (Fig. 1) y curva enzimática compatible con necrosis miocárdica (troponina: $4.52 \mathrm{ng} / \mathrm{mL}$ ). En el ecocardiograma transtorácico (ETT) practicado urgentemente se apreció una acinesia apical extensa junto a un gradiente intraventricular superior a $90 \mathrm{mmHg}$ e insuficiencia mitral (IM) moderadasevera. La paciente recibió tratamiento antiagregante y betabloqueante, desestimándose la realización de fibrinolisis, pues contaba con más de 12 horas de evolución. Tras ello, permaneció asintomática. Pasados tres días de evolución apareció en el ECG una isquemia subepicárdica anterolateral extensa (Fig. 1) por lo que se solicitó una

Trabajo aceptado: 22 de diciembre de 2005

Correspondencia: Blanca Obón Azuara. Servicio de Medicina Interna. Hospital Clínico Universitario. Avda. S. Juan Bosco, 15. 50009 Zaragoza. E-mail: blankaobona@yahoo.es 


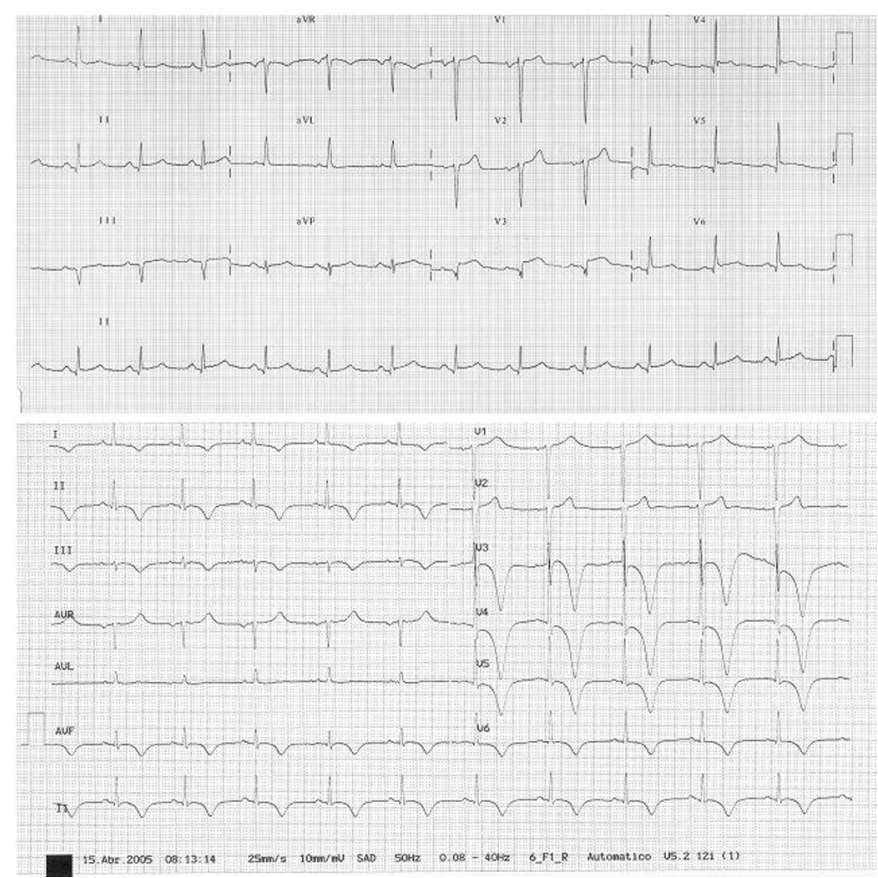

Fig. 1. ECG basal durante el episodio agudo del SDAT con elevación del ST en serie precordial e inferior (parte superior de la imagen) y su evolución a isquemia subepicárdica (parte inferior de la imagen).

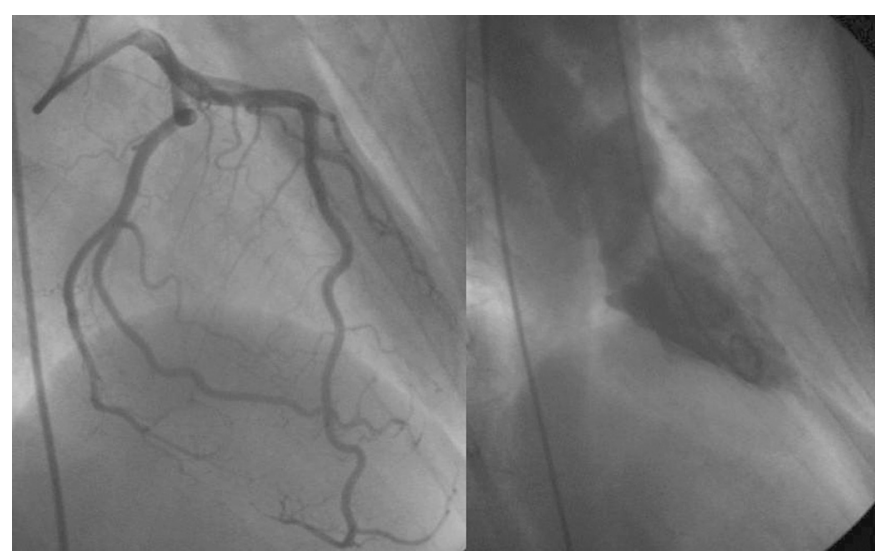

Fig. 2. Cateteterismo cardiaco diagnóstico tras el episodio agudo de SDAT. Ventriculografía en sistóle (izquierda), y coronariografía con dominancia izquierda, sin lesiones angiográficas (derecha).

coronariografía donde las arterias coronarias no mostraban lesiones angiográficas, el ventrículo izquierdo (VI) no se encontraba hipertrofiado, su contractilidad segmentaria estaba conservada y no existía gradiente en el TSVI (Fig. 2). Fue dada de alta con tratamiento antiagregante, betabloqueante e hipolipemiante. La evolución posterior fue favorable, y a los dos meses del ingreso se retiró el tratamiento betabloqueante en la consulta de cardiología.

Transcurrido un mes desde dicha consulta, la paciente sufre de nuevo, tras otra discusión, un episodio de dolor torácico similar al de hacía unos meses aunque de menor intensidad, motivo por el que ingresó en la UCI de nuestro hospital. El ECG era compatible con la normalidad y no existió elevación enzimática. En la exploración física se auscultó un soplo pansistólico irradiado a axila y a carótidas, por lo que se realizó un ETT urgente en el que se apreció un VI no

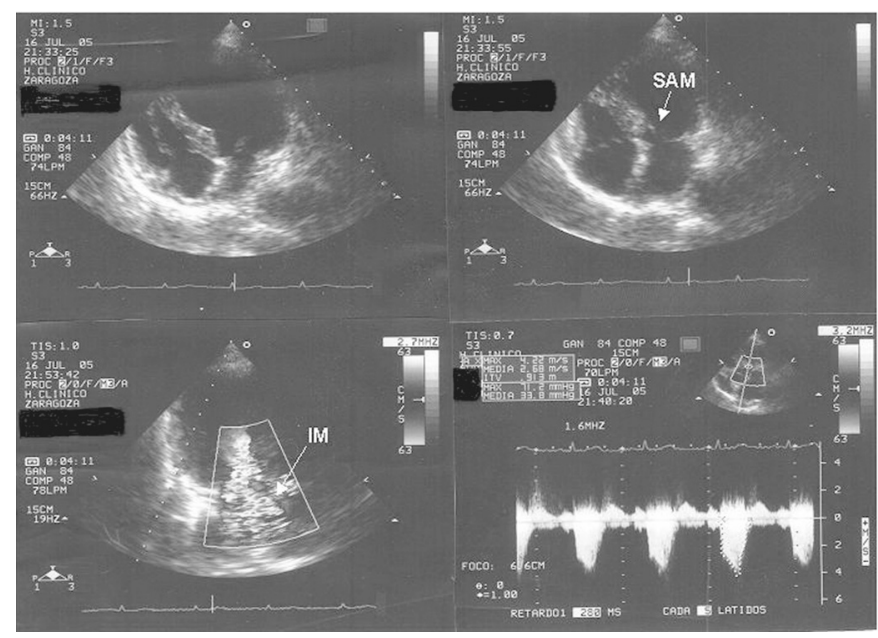

Fig. 3. Ecocardiograma transtorácico en un plano apical de 4 cámaras durante el $2^{\circ}$ episodio donde se aprecia la geometría del TSVI con SAM (parte superior de la imagen), IM significativa y gradiente elevado en TSVI (parte inferior de la imagen).

hipertrofiado con un TSVI estrecho y rodilla septal prominente que condicionaba un gradiente máximo de hasta $70 \mathrm{mmHg}$, movimiento sistólico del velo anterior (SAM) e IM secundaria III-IV (Fig. 3) que invertía parcialmente el flujo sistólico en venas pulmonares. La contractilidad global y segmentaria estaba conservada y la aurícula izquierda era pequeña. Ante la sospecha de obstrucción dinámica severa en TSVI y teniendo en cuenta los antecedentes descritos, se inició tratamiento betabloqueante de modo precoz y a altas dosis, tras el que la paciente permaneció asintomática. Transcurridas doce horas un segundo ETT mostró la desaparición tanto de gradiente significativo a nivel de TSVI (gradiente máximo $16 \mathrm{mmHg}$ ), como del SAM, y la permanencia de una IM II/IV.

\section{DISCUSIÓN}

El presente caso se trata de una paciente postmenopáusica que tres meses antes había sufrido un SDAT tratado en otro hospital. Tras una evolución inicial favorable y coincidiendo con la ausencia de tratamiento betabloqueante, la paciente sufre de nuevo un cuadro de estrés emocional que le reproduce la sintomatología previa, siendo remitida a nuestro hospital donde, tras el diagnóstico inicial e instauración del tratamiento betabloqueante, se logra tanto la desaparición de la clínica como del gradiente en TSVI.

Nos encontramos ante una recidiva de un SDAT en esta ocasión "abortado", en la que la falta de intensidad del evento emocional (con menor descarga adrenalínica) junto con la rapidez de instauración del tratamiento betabloqueante impidieron la evolución a una isquemia apical grave, en una paciente con antecedente de SDAT y gradiente en TSVI. La historia de un cuadro clínico similar y la existencia de un gradiente dinámico significativo en un TSVI, de geometría característica, no presente en la coronariografía diagnóstica y que además desaparece tras iniciar tratamiento betabloqueante, concuerda con este diagnóstico cuyo mecanismo patógeno inicial sería la existencia de un gradiente dinámico en el TSVI $(5,6)$. 


\section{Bibliografía}

1. Tsuchihaschi k, Ucshima K, Uchida T, Oh-umma $\mathrm{N}$, Kiuma K, Owa M, et al. Trasient left ventricular apicla ballooning without coronary artery stenosis a novel heart syndrome mimicking acute myocardial infarction. Angina Pectoris Myocardial Infarction Investigations in Japan. J Am Coll Cardiol 2001; 38: 11-8

2. Bybee KA, Kara T, Prasad A, Lerman A, Barsness GW, \& al. Systematic Review: Transient Lewft Ventricular Apical Ballooning: a syndrome that mimics ST-segment elevation myocardila infarction. Ann Intern Med 2004; 141: 858-865.

3. Ferrer Gracia MC, Ortas Nadal MR, Daga Calejero B. Dolor torácico y fibrilación ventricular con coronarias sanas y abalonamiento apical izquierdo transitorio. Med Clin 2004; 123: 52.
4. Gallego Page JC, Lafuente Gormaz C, Domínguez Rodríguez P, Cháfer Rudilla $\mathrm{M}$, Fuentes Manso R, Aguilera Saldaña M. Disfunción ventricular transitoria tras estrés emocional. Rev Esp Cardiol 2004; 57 (11): 1124-7.

5. Barriales Villa R, Bilao Quesada R, Iglesias Río E, Bayón Meleiro N, Mantill González R, Penas Lado M. Síndrome de discinesia apical transitoria sin lesiones coronarias. Importancia del gradiente intraventricular. Rev Esp Cardiol 2004; 57: 85-88.

6. Penas-Lado M, Barriales-Villa R, Goicochea J. Trasient left ventricular apical ballooning and outflow tract obstruction. J Am Coll Cardiol 2003; 42: 1143-4. 